\title{
Explore common Achilles tendon injuries in
}

\section{runners Lauren Gowland, David Knowles BSc MB BChir FRCS}

\section{INTRODUCTION AND BACKGROUND}

The incidence of Achilles tendon injuries in runners is approximately 10 times that in age matched controls. ${ }^{1}$ The Achilles tendon is the thickest tendon in the human body. It attaches the plantaris, gastrocnemius and soleus muscle to the posterior tuberosity of the calcaneus serving its main function to plantar flex the foot. ${ }^{2}$ It is surrounded by a connective tissue sheath also known as a paratendon. This structure is highly vascular and allows the tendon to glide more easily with movement. ${ }^{3}$

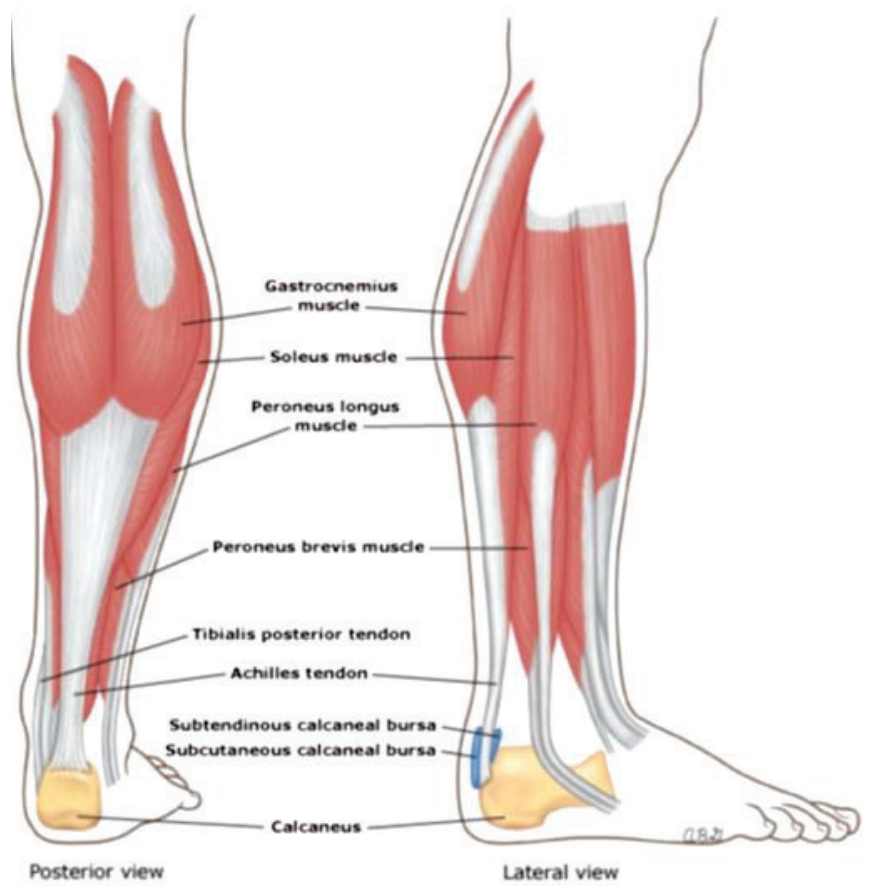

Anatomy of the Achilles tendon ${ }^{4}$

Specific injuries of the Achilles tendon to be discussed in this report include Achilles tenosynovitis, Achilles tendinitis, Achilles tendinosis, Achilles rupture and Achilles tendinopathies, which can be categorised further based on anatomical location into insertional and noninsertional tendinopathy. For clarification purposes, the definitions and comparative factors of the main Achilles tendon injuries are detailed below.

Achilles tenosynovitis is inflammation of the sheath surrounding the tendon. It commonly causes fibrosis and scarring within the sheath restricting the range of motion of the Achilles tendon. Symptoms experienced with this condition include pain, tender swelling around the Achilles and crepitus on movement of the ankle joint. Causes for this condition relate to having a tight calf and Achilles complex, changing the volume of training completed or changing the type of footwear being worn. ${ }^{5}$

Management options for Achilles tenosynovitis can be divided into short and long term plans. Firstly, rest from the aggravating sport. Secondly, at the time of the injury, ice and compression should be applied for approximately 10 to 15 minutes every hour for 3 to 4 hours. This will ease pain along with reducing swelling and inflammation. Non-steroidal anti-inflammatory drugs may also be given for a few days to a week to reduce inflammation. Longer term management of Alfredson's eccentric heel drops, massage and a change in footwear is recommended.

Achilles tendinopathy is an umbrella term for clinical conditions in and surrounding the tendon. Only once a histological sample has been studied may the term tendinosis and tendinitis be applied. Histologically a tendinopathy is a non-inflammatory intra-tendinous collagen degeneration with fibre disorientation and thinning. The tendon will likely show scattered vascular ingrowth, hypercellularity and increased interfibrillar glycosaminoglycans. ${ }^{1}$ The overall bulk of the Achilles tendon will increase whilst its overall strength will decrease.

The two types of tendinopathy include insertional, where the Achilles joins to the calcaneus and noninsertional, approximately two to six centimetres proximal to this point. ${ }^{6}$ The main symptoms associated with this condition include increasing pain, stiffness and crepitus around the tendon, similar to that seen in tenosynovitis. Heat and light walking are known to improve the symptoms whereas strenuous activity is known to exacerbate the injury. Marked tenderness in the surrounding areas especially over the mid-point of the tendon, the most common type of Achilles tendinopathy, is a common symptom experienced. ${ }^{7}$

The failed healing response of tendinopathy has three different and continuous stages. These comprise of reactive tendinopathy, tendon disrepair and degenerative tendinopathy. ${ }^{6}$ Firstly, reactive tendinopathy is a short term response whereby the Achilles thickens and stiffens in an attempt to reduce the load being placed upon it. The tendon disrepair is due to delineation of the tendon fibres. Finally, in the degenerative tendinopathy stage, which is more common in older athletes, due to chronic overloading, collagen becomes disorganized and the matrix begins to breakdown. Increased vascularity and neuronal ingrowth contribute to this stage. ${ }^{8}$

Achilles tendinosis is a degenerative process of the tendon with little histological or clinical signs of intratendinous inflammation. ${ }^{9}$ There are many theories in regards to the aetiology of this condition. For example, overuse of the Achilles, muscle imbalance or weakness, decreased blood supply and tensile strength with aging or even malalignment such as hyper-pronation. ${ }^{10,} 11$ The tenocytes themselves produce substance $\mathrm{P}$ and prostaglandin E2 inflammatory mediators, in response to increased loading. ${ }^{6}$ This subsequently leads to delineation of the tendon fibres.

Achilles tendinitis is inflammation of the Achilles tendon itself. There are two main locations where this occurs, firstly, at the insertion point where the tendon attaches to the calcaneus and secondly, at the mid-point of the tendon. In both cases the damaged tendon fibres may calcify providing symptoms of stiffness and pain. There have been numerous studies conducted to determine the best treatment outcomes for Achilles tendinitis however the aetiology and treatment still remains unclear. ${ }^{12}$ 
In terms of Achilles tendon rupture, the incidence in the UK is approximately 11,000 per year. ${ }^{13}$ The mechanism of injury is usually acute with a bi-modal age distribution for incidence, the first being athletes between 30-40 years old, who participate in any sport which requires an eccentric muscle contraction such as a forceful 'push off' from the foot. For example, football, sprinting, diving and tennis. ${ }^{3}$ The second being the age range of 60 80 years old who rupture their Achilles doing everyday tasks such as climbing the stairs. ${ }^{13}$ The most common symptom the patient describes is a clear popping sensation followed by the diminished ability to weight bear. The foot is commonly in the dorsiflexed position when this occurs $^{14}$. Risk factors for Achilles tendon rupture include increasing age, recurrent Achilles tendinopathy, systemic corticosteroids and systemic conditions such as gout, rheumatoid arthritis and systemic lupus erythematous. ${ }^{3}$ The area ruptured most commonly is approximately two inches above the calcaneal attachment due to a zone of avascularity. ${ }^{2}$

Diagnosing Achilles tendon pathology in runners can be aided by the use of ultrasound machines, $\mathrm{X}$-rays or MRI machines. ${ }^{15}$ The most commonly used method is via ultrasound as the machines are commonly available, safe to use and appropriately provide the necessary information about the Achilles tendinopathy diagnosis. ${ }^{16}$
Three main aims are to be addressed in this report. Firstly, to differentiate between common Achilles tendon injuries experienced by runners; secondly, to discuss the guidelines recommended by key bodies such as National Institute for Health and Care Excellence (NICE) for the management of Achilles tendinopathy and rupture; and thirdly, to research studies relevant to that of Achilles tendinopathies to provide evidence to support management options. Finally, the report will discuss potential limitations of management options for Achilles injuries and summarise key recommendations.

\section{DISCUSSION}

Achilles tendinopathy is a common injury experienced by athletes whose sport requires them to run. Sixty-six percent of Achilles tendon problems occur in joggers which emphasizes the role running has in the aetiology of such injuries. ${ }^{12}$ It is characteristically chronic overuse of the Achilles tendon whilst training. Other causes of tendinopathy include extreme overloading such as rapid increase in training volume, poorly fitting footwear, change in running surface or excessive pronation of the foot. ${ }^{3}$ Medical reasons that may contribute to tendinopathy include hypertension, diabetes, familial hypercholesterolemia and the use of quinolone antibiotics ${ }^{3}$.

\begin{tabular}{|c|c|c|c|c|}
\hline $\begin{array}{l}\text { Pathologic } \\
\text { diagnosis }\end{array}$ & $\begin{array}{c}\text { Concept } \\
\text { (macroscopic } \\
\text { pathology) }\end{array}$ & $\begin{array}{c}\text { Physical } \\
\text { examination }\end{array}$ & Imaging & Histopathology \\
\hline Tendinosis- & $\begin{array}{l}\text { Intratendinous } \\
\text { degeneration }\end{array}$ & $\begin{array}{l}\text { Tendon } \\
\text { thickening and } \\
\text { tenderness on } \\
\text { palpation of the } \\
\text { midportion }\end{array}$ & $\begin{array}{l}\text { Hypoechoic areas on grey-scale } \\
\text { ultrasonography; increased tendon } \\
\text { thickness; increased colour Doppler } \\
\text { signal, usually originating from deep } \\
\text { surface }\end{array}$ & $\begin{array}{l}\text { Collagen disorientation. disorganization and fibre } \\
\text { separation; hypervascularity; variable tenocyte density } \\
\text { (usually increased fibroblastic and myofibroblastic } \\
\text { cellularity); evidence of microtears (fibrin, hemosiderin) }\end{array}$ \\
\hline Paratendonitis- & $\begin{array}{l}\text { Inflammation of the } \\
\text { outer layer of the } \\
\text { tendon (paratendon) }\end{array}$ & Crepitus & Paratendinous edema & $\begin{array}{l}\text { Mucoid degeneration and scattered inflammatory cells in } \\
\text { the paratendon }\end{array}$ \\
\hline $\begin{array}{l}\text { Paratendonitis } \\
\text { with } \\
\text { tendinosis }\end{array}$ & $\begin{array}{l}\text { Paratendonitis } \\
\text { associated with } \\
\text { intratendinous } \\
\text { degeneration }\end{array}$ & $\begin{array}{l}\text { Combinations of } \\
\text { the above }\end{array}$ & Combinations of the above & $\begin{array}{l}\text { Degenerative changes as noted in tendinosis, with mucoid } \\
\text { degeneration, with or without fibrosis, and scattered } \\
\text { inflammatory cells in the paratendon }\end{array}$ \\
\hline Partial rupture & $\begin{array}{l}\text { Macroscopic defect of } \\
\text { collagen fascicles }\end{array}$ & $\begin{array}{l}\text { Tendon } \\
\text { thickening: pain } \\
\text { on stretch: } \\
\text { marked weakness }\end{array}$ & $\begin{array}{l}\text { Collagen defect on grey-scale } \\
\text { ultrasonography; increased colour } \\
\text { Doppler signal, usually on superficial } \\
\text { surface }\end{array}$ & $\begin{array}{l}\text { Evidence of tearing. inflammation and granulation tissue; } \\
\text { adjacent areas often show tendinosis }\end{array}$ \\
\hline $\begin{array}{l}\text { Complete } \\
\text { rupture }\end{array}$ & $\begin{array}{l}\text { Complete loss of } \\
\text { continuity of tendon } \\
\text { fibres }\end{array}$ & $\begin{array}{l}\text { Lack of strength } \\
\text { on plantar } \\
\text { flexion; Homan } \\
\text { sign }\end{array}$ & Obvious defect on ultrasonography & $\begin{array}{l}\text { Evidence of tearing. inflammation and granulation tissue: } \\
\text { adjacent areas often show tendinosis }\end{array}$ \\
\hline $\begin{array}{l}\text { Insertional } \\
\text { disorders }\end{array}$ & $\begin{array}{l}\text { Injury, inflammation } \\
\text { or degeneration of the } \\
\text { osteotendinous } \\
\text { junction, bursae or } \\
\text { both }\end{array}$ & $\begin{array}{l}\text { Tendon } \\
\text { thickening and } \\
\text { tenderness on } \\
\text { palpation of the } \\
\text { insertional area }\end{array}$ & $\begin{array}{l}\text { Hypoechoic areas or defects adjacent to } \\
\text { the calcaneus; bursitis; defects of the } \\
\text { calcaneus }\end{array}$ & Not available \\
\hline
\end{tabular}

Comparison of common Achilles tendon pathologies and clinical findings ${ }^{14}$ 
A positive association between Achilles tendinopathy and family history has also been established. ${ }^{17}$

The treatment of Achilles tendinopathies is multifactorial and highly controversial. According to NICE, ${ }^{27}$ first line management for Achilles tendinopathies includes rest; stopping the precipitating sport that caused the injury and to provide analgesia for the pain itself. Nonsteroidal anti-Inflammatory drugs (NSAIDs) are advised for an acute injury but longer term paracetamol would be preferable due to the lower risk of side effects. In terms of easing symptoms, the application of ice packs or cold compresses to the area immediately after the injury will be of some benefit.

Evidence proves that eccentric rehabilitation programmes for the Achilles tendon promote the most effective outcomes. An eccentric exercise is one where the muscle or tendon lengthens as it contracts. The Achilles responds to this type of exercise as it is argued the fibres of the tendon are reorganised to be able to deal with a higher load. Therefore this allows the Achilles to become stronger. $^{18}$

A meta-analysis of 11 different randomized controlled trials suggested that a patient's pain, overall function and satisfaction improved after 12 weeks of eccentric exercise. ${ }^{19}$ Long term, after five years, sustained results were experienced by the patients who had completed Alfredson's protocol. ${ }^{20}$ These results support the use of eccentric rehabilitation exercises in Achilles tendinopathies as opposed to concentric based exercise programmes or mixed programmes. ${ }^{21}$ An example of a mixed programme is that of Silbernagel's. He varies eccentric exercises with concentric exercises once a day for 12 weeks. However his results aren't as beneficially globally perceived as Alfredson's purely eccentric programme. ${ }^{22}$

One of the recommended exercise programmes based on eccentric heel drops was devised in 1998 by Dr Hakan Alfredson, a Swedish sports medicine doctor. It is known as "Alfredson's heel drop protocol". The exercise regime comprises three sets of 15 heel drops twice daily for 12 weeks. ${ }^{23}$

The exact technique to perform these heel drop exercises involves the patient standing on a step with both heels overhanging. The patient lifts the injured heel off the step and raises the good heel to plantarflex the foot, still on the step. Secondly, the weight is transferred onto the injured foot in the plantar flexed position, over the edge of the step. Finally, to complete the exercise, the heel is slowly lowered over the edge of the step over approximately 10 seconds until the foot is in the dorsiflexed position. ${ }^{24}$ There are two variations to this exercise depending on how much load the tendon can withstand. Described above is the first whilst the second option is to bend the injured leg. This puts more weight through the tendon to strengthen it further.

No training programme goes without its limitations. The first one being that Alfredson's quantity of advised exercises is overly time-consuming; managing up to 120 180 heel drops per day. It was noted that throughout the length of the training programme compliance rates dropped to $50 \%$ by the 12 th week. ${ }^{26}$ Secondly, symptoms may worsen before any improvements are seen. ${ }^{3}$ This may cause numerous participants to stop the exercises if efficient communication has not been provided to inform them of this. Another limitation is that relating to elderly patients who are practically immobile; they will not be able to withstand their own weight on the edge of a step to complete the exercises.

Other potential management options for Achilles tendinopathies include orthotic devices which are to be worn in shoes at all times. This will aid runners who have
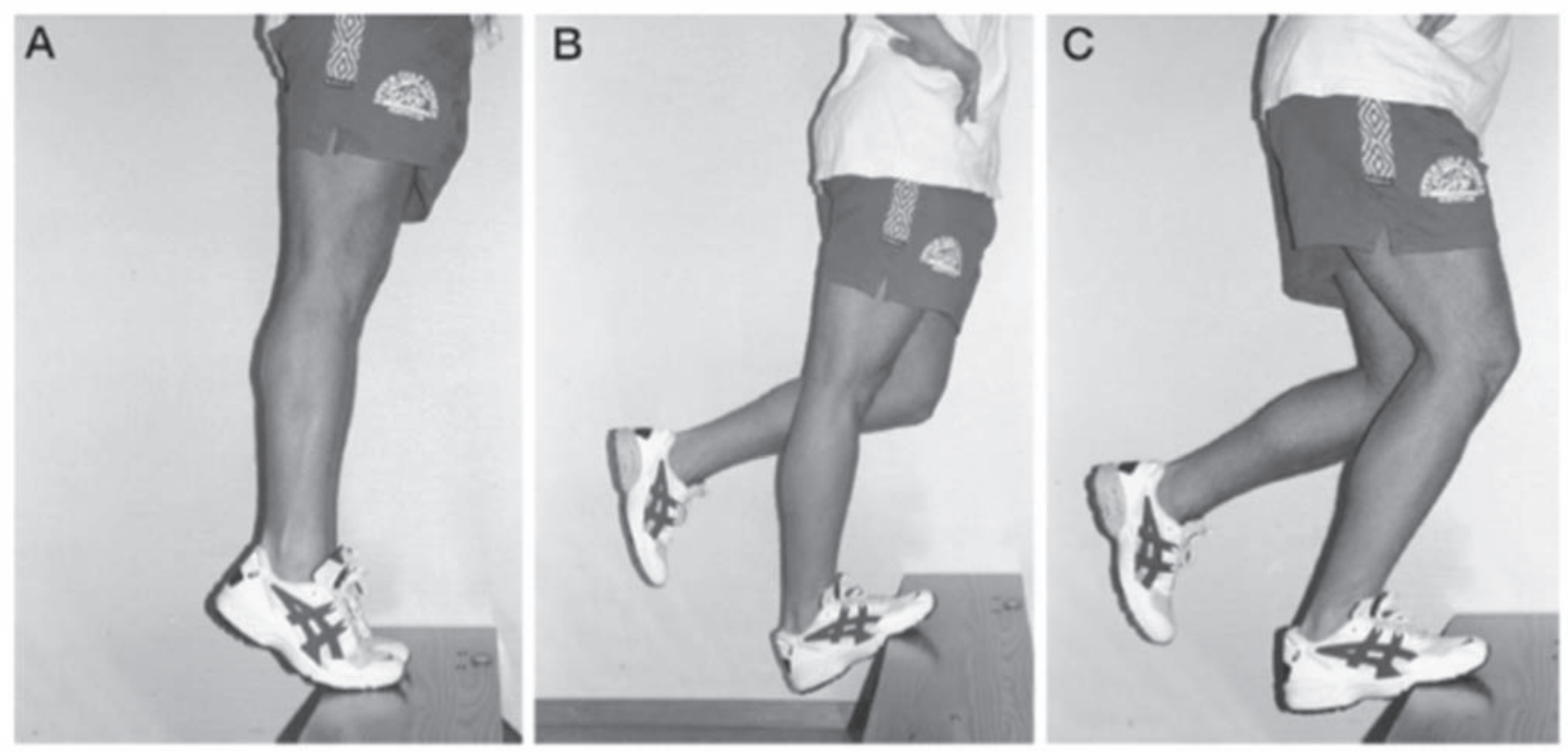

Figure 1. From an upright body position and standing with all body weight on the forefoot and the ankle joint in plantar flexion lifted by the noninjured leg (A), the calf muscle was loaded eccentrically by having the patient lower the heel with the knee straight $(B)$ and with the knee bent $(C)$.

Diagram showing correct technique of Alfredson's heel drop rehabilitation programme ${ }^{25}$ 
an over-pronated running strike. The aim is to marginally lift the heel or help support the arch of the foot both with the aim of reducing the strain on the Achilles tendon whilst it heals.

Non-medical management options for Achilles tendon injuries involve changing the running surface. For example, run on grass or trail instead of road to reduce the load put through the Achilles tendon. Similarly, reassess the choice of footwear. Many runners who suffer with Achilles tendinopathies have a hyper-pronated running style; this is the movement of the subtalar joint into eversion, dorsiflexion and abduction. Finding a supportive shoe to help counteract some of this movement will reduce the susceptibility to such injuries.

In terms of treatment provided by secondary care, $\mathrm{NICE}^{27}$ does not support the use of steroid injections as a recognised treatment for Achilles tendinopathies. This is due to the increased risk of tendon atrophy or rupture proven by a recent Cochrane review. ${ }^{9}$ They do however offer extracorporeal shock wave therapy (ESWT). Acoustic shockwaves are passed through the skin and directed at the Achilles tendon; the main aim of this treatment is to increase blood flow to the area and promote regrowth of the tendon in a linear fashion. Another similar technique is the use of low level laser therapy; this method is thought to increase collagen production and reduce blood flow in new vessels. ${ }^{27}$ Both of these management options are best used as adjuncts to eccentric Achilles exercises.

Non operative methods of Achilles tendon repair include functional bracing or casting in resting equinus. This management method would be more likely considered in medically frail patients who are sedentary for considerable periods of time. An advantage of using this method is that there are fewer complications to that of an operative approach. Also, there is argued to be equivalent plantar flex strength compared to operative procedures after recovery. However, choosing the nonoperative management method increases the chance that the Achilles tendon may re-rupture. ${ }^{28}$

Rarely, resistant Achilles tendinopathy may require open surgery to excise fibrous adhesions and degenerative nodules. ${ }^{27}$ An indication for this method is if the initial injury occurred less than six weeks previously. Another option for Achilles surgery is percutaneous Achilles tendon repair; however the disadvantage of using this method is damage to local structures. Consequently, there is an increased risk of sural nerve damage. For chronic ruptures with a defect of less than approximately three centimetres, a $\mathrm{V}-\mathrm{Y}$ advancement technique should be considered. If the patient has a functioning tibial nerve and a rupture of more than three centimetres then a flexor hallucis longus transfer with or without a V-Y advancement of gastrocneumius may be the best option. ${ }^{28}$

On deciding the most appropriate treatment option the risk benefit analysis needs to be considered. For example, if casting for a 12 week period was chosen over open surgery, the risk of deep vein thrombosis (DVT) would be greater due to immobility for that time. Similarly, choosing a non-operative approach would increase the chance of joint stiffness after recovery. The treatment options need to be considered on an individual basis to ensure the best quality of life for each patient.
With any surgery the general complications have to be taken into consideration. For example bleeding, infection, anaesthetic risk, failure of operation and wound breakdown. More specific to Achilles surgery, complications include scar and pain around the tendon and injury to surrounding structures being the sural nerve and posterior tibial artery. ${ }^{29}$

\section{SUMMARY}

The current guidance recommended by NICE on management of Achilles tendinopathy ${ }^{27}$ supports the exercise rehabilitation programme with evidence for the best outcomes. This is that of Alfredson's 12 week programme of eccentric heel drops. Evidence supports that this programme is noted to reduce pain and improve symptoms within this patient cohort. ${ }^{20}$ This strengthens the recommendation that patients should undergo a purely eccentric exercise programme if general mobility allows them.

Surgical methods of treatment are of main benefit in acute rupture as opposed to tendinopathies. Younger athletes with full rupture are more likely to benefit from surgical repair as risk of re-rupture is reduced. The risk of DVT is also reduced as mobility after surgery is increased. With a possible future sporting career the benefits of surgery are likely to outweigh any risk.

Overall it can be concluded that there are numerous Achilles tendon injuries that runners can experience. With the correct diagnosis and appropriate management measures put in place, recovery will usually take around 12 weeks. As the treatment options available for Achilles tendinopathy are so vastly controversial the risks and benefits of each option need to be thoroughly assessed and matched to each individual case before a final decision is made. ${ }^{6}$ The current management guidelines issued by $\mathrm{NICE}^{27}$ have good supporting evidence for positive outcomes. The options and evidence assessed through this paper lead to the conclusion that no changes to current guidelines should be recommended.

\section{REFERENCES}

1. Maffulli N, Sharma P, Luscombe KL. Achilles tendinopathy: aetiology and management. Journal of the Royal Society of Medicine. 2004;97(10):472-6.

2. Wikipedia. Achilles tendon. Wikipedia 2017 [updated 3rd September 2017].Available at: https:// en.wikipedia.org/wiki/Achilles_tendon (accessed 20.9.17)

3. Payne J. Achilles tendinopathy and rupture. Patient. co.uk; 2016 [updated 2nd June 2016] Available at: https://patient.info/doctor/achilles-tendinopathyand-rupture (accessed 2.6.17).

4. Human Anatomy Library. Achilles Tendon Anatomy Diagram; 2016 [updated 7th July 2016]. Available at: http://humananatomylibrary.com/achilles-tendonanatomy-diagram/ (accessed on 25.10.17).

5. Kerkar P. Achilles tenosynovitis or paratenonitis: causes: symptoms, treatment, recovery time, exercises. Pain Assist Inc; 2018 [updated 3rd January 2018]. Available at: https://www.epainassist.com/ sports-injuries/ankle-injuries/achilles-tenosynovitis (accessed 25.1.18). 
6. Li H-Y, Hua Y-H. Achilles tendinopathy: current concepts about the basic science and clinical treatments. BioMed Research International. 2016;2016:6492597.

7. McCormack JR. The management of mid-portion achilles tendinopathy with Astym $\AA$ and eccentric exercise: a case report. International Journal of Sports Physical Therapy. 2012;7(6):672-7.

8. Fu S-C, Rolf C, Cheuk Y-C, Lui PP, Chan K-M. Deciphering the pathogenesis of tendinopathy: a three-stages process. Sports Medicine, Arthroscopy, Rehabilitation, Therapy \& Technology: SMARTT. 2010;2:30-.

9. Lopez RG, Jung HG. Achilles tendinosis: treatment options. Clinics in Orthopedic Surgery. 2015;7(1):17. Epub 2015/03/03.

10. Lopez RGL, Jung H-G. Achilles tendinosis: treatment options. Clinics in Orthopedic Surgery. 2015;7(1):17.

11. Lake JE, Ishikawa SN. Conservative treatment of Achilles tendinopathy: emerging techniques. Foot Ankle Clin. 2009;14(4):663-74.

12. Almekinders LC, Temple JD. Etiology, diagnosis, and treatment of tendonitis: an analysis of the literature. Medicine and Science in Sports and Exercise. 1998;30(8):1183-90. Epub 1998/08/26.

13. Costa M. UK STAR: UK Study of tendo Achilles Rehabilitation -multicentre randomised clinical trial. Available at: https://ukstar.octru.ox.ac.uk/ (accessed 1.3.18)

14. Khan KM, Cook JL, Bonar F, Harcourt P, Astrom M. Histopathology of common tendinopathies. Update and implications for clinical management. Sports medicine. 1999;27(6):393-408. Epub 1999/07/27.

15. Sode J, Obel N, Hallas J, Lassen A. Use of fluroquinolone and risk of Achilles tendon rupture: a population-based cohort study. European Journal of Clinical Pharmacology. 2007;63(5):499-503. Epub 2007/03/06.

16. Maffulli N, Regine R, Angelillo M, Capasso G, Filice S. Ultrasound diagnosis of Achilles tendon pathology in runners. British Journal of Sports Medicine. 1987;21(4):158-62.

17. Kraemer R, Wuerfel W, Lorenzen J, Busche M, Vogt PM, Knobloch K. Analysis of hereditary and medical risk factors in Achilles tendinopathy and Achilles tendon ruptures: a matched pair analysis. Archives of Orthopaedic and Trauma Surgery. 2012;132(6):84753. Epub 2012/02/22.

18. Groom T. Achilles Tendinopathy. Running Physio; 2012 [updated 6th April 2012]. Available at: http:// www.running-physio.com/achilles-tendinopathy/ (accessed 26.9.17).

19. Asplund CA, Best TM. Achilles tendon disorders. BMJ;2013;346.

20. van der Plas A, de Jonge $\mathrm{S}$, de Vos RJ, van der Heide HJL, Verhaar JAN, Weir A, et al. A 5-year follow-up study of Alfredson's heel-drop exercise programme in chronic midportion Achilles tendinopathy. British Journal of Sports Medicine. 2012;46(3):214-8.

21. Mafi N, Lorentzon R, Alfredson H. Superior shortterm results with eccentric calf muscle training compared to concentric training in a randomized prospective multicenter study on patients with chronic Achilles tendinosis. Knee Surgery, Sports Traumatology, Arthroscopy. 2001;9(1):42-7. Epub 2001/03/28.

22. Habets B, van Cingel REH, Backx FJG, Huisstede BMA. Alfredson versus Silbernagel exercise therapy in chronic midportion Achilles tendinopathy: study protocol for a randomized controlled trial. BMC musculoskeletal disorders. 2017;18(1):296. Epub 2017/07/12.

23. Brockerville M. Achilles Tendinopathy:Current Concepts in Treatment and Rehabilitation. Athlete's Edge. Available at: http://www.wsm.ca/files/www/ Athletes_Edge/AE_Achilles_handout.pdf (accessed 1.3.18).

24. Sports Injury Clinic. Achilles tendonitis exercises. 2017 [updated 2017]. Available at: http://www. sportsinjuryclinic.net/sport-injuries/ankle-achillesshin-pain/achilles-tendonitis/strengtheningexercises-achilles-tendinitis. (accessed on 26.9.17).

25. Writings R. Achilles tendonitis in runners: A degenerative overuse injury best treated with eccentric heel drops. Running Writings; 2013 [updated 19th November 2013]. Available from: http://www. runningwritings.com/2013/11/achilles-tendonitisin-runners.html (accessed on 15.10.17).

26. Roos EM, Engstrom M, Lagerquist A, Soderberg B. Clinical improvement after 6 weeks of eccentric exercise in patients with mid-portion Achilles tendinopathy -- a randomized trial with 1-year followup. Scandinavian Journal of Medicine \& Science in Sports. 2004;14(5):286-95. Epub 2004/09/25.

27. NICE (2016). Achilles tendinopathy. Available at: https://cks.nice.org.uk/achilles-tendinopathy\#! scenario (accessed on 26.9.17).

28. Karadsesh R. Achilles tendon rupture. Orthobullets; 2015. Available at: https://www.orthobullets.com/ foot-and-ankle/7021/achilles-tendon-rupture (accessed 4.10.17).

29. Ahmed IM, Lagopoulos M, McConnell P, Soames RW, Sefton GK. Blood supply of the Achilles tendon. Journal of Orthopaedic Research. 1998;16(5):591-6. Epub 1998/11/20.

Correspondence to: Lauren Gowland l.gowland@lancaster.ac.uk 